The Impact of Large-Scale Surveys on Pulsating Star Research

ASP Conference Series, Vol. 203, 2000

L. Szabados \& D. W. Kurtz, eds.

\title{
Changes of the Physical State in Semiregular Variables
}

\author{
L. L. Kiss, Gy. Szabó, K. Szatmáry
}

Department of Experimental Physics \& Astronomical Observatory, JATE University, H-6720 Szeged, Dóm tér 9., Hungary

\section{J. A. Mattei}

AAVSO, 25 Birch Street, Cambridge MA02138-1205, USA

\begin{abstract}
We present a progress report on a detailed analysis of longterm (70-90 years) visual observations for seven semiregular variables. Fundamental changes of the physical state (amplitude and/or frequency modulations, mode change and switching) are studied with conventional Fourier- and wavelet analyses. Besides two examples of repetitive mode changes we report a simple geometric model of a rotationally induced amplitude modulation in RY Ursae Majoris.
\end{abstract}

\section{Introduction}

Semiregular stars of type SRa and SRb are pulsating red variables on the asymptotic giant branch (AGB) of the Hertzsprung-Russell diagram. The attribute "semiregular" refers to the complexity of their visual light curves, which usually takes the form of cycle-to-cycle variations in a relatively simple pulsating environment. There has been an increasing amount of empirical evidence that multiperiodicity occurs is some stars (e.g. Kiss et al. 1999), which suggests the existence of many simultaneously excited modes of pulsation.

From the observational point of view, the main parameters characterizing the pulsation are as follows: the number of excited modes, their orders (in case of overtone pulsation), periods and amplitudes. Fundamental changes of these parameters (e.g. mode-switching, repetitive turning on and off of different modes, long-term amplitude or frequency modulations) may indicate incomplete understanding of the physical processes governing the pulsation and stellar evolution.

\section{Observations and Results}

This analysis is based on long-term (70-90 years) visual observations for 7 stars (V Boo, W Cyg, RU Cyg, TX Dra, Y Per, RX UMa and RY UMa) carried out by amateur astronomers. Newly computerized historic and very recent data, collected by the AAVSO and VSNET, have been added to the earlier observations (see Kiss et al. 1999) taken from the international databases of the Association Française des Observateurs d'Etoiles Variables (AFOEV), the Variable Star Observers' League of Japan (VSOLJ), and the Hungarian Astronomical 
Association/Variable Star Section (HAA/VSS). The data were analyzed with conventional Fourier and wavelet analyses.

Y Per, RU Cyg and V Boo illustrate the long-term amplitude decrease, also observed in $\mathrm{R}$ Dor (Bedding et al. 1998). Here we discuss the possibility that the type of variability might be changing (from Mira or SRa to SRb) simultaneously with the appearance of a new mode. W Cyg and TX Dra are the most illustrative examples of repetitive mode changes, where some modes turn on and off on a time scale of few thousands of days. One of the most striking features is the abruptness of mode changes. A possible explanation is a strong convectionpulsation coupling, as the time scales of mode change and convection are of similar orders of magnitude, and the extended convective envelopes of these stars are well-suited places for such a connection (Xiong, Ding, \& Cheng 1998).

RU And, RX UMa and RY UMa show very interesting amplitude modulations. The light curves of RU And and RX UMa can be described with two closely separated periods, which are physically reasonable (1st plus 3rd/4th overtones), but RY UMa is a different case. One factor which has generally been neglected in earlier studies is stellar rotation, because typical rotational periods of red giants (4000-10000 days) are much longer than the characteristic times of pulsation. However, RY UMa shows such complex light variations and frequency spectrum that a possible explanation could be the rotation-pulsation connection. The highly repetitive amplitude modulation of RY UMa $\left(P_{\bmod }=16 \cdot P_{\text {pul }}\right.$ $=4900$ days $)$ closely resembles the Blazhko-effect in RR Lyrae variables. We build a very simple model, which involves a rotationally modulated nonradial oscillation. The impressive qualitative agreement may suggest the possibility of a highly distorted stellar shape, which has already been observed in some Mira variables. Asida \& Tuchman (1995) explored the asymmetrical mass-loss from rotating red giant variables and presented a scenario for an anisotropic mass ejection from AGB variables caused by rotation effects. We note that Barnbaum, Morris, \& Kahane (1995) have suggested a possible connection between rapid rotation and pulsation in V Hya (type SRa), while high-resolution ground-based observations and direct imaging with the Hubble Space Telescope have revealed non-spherical structures in some Mira-type variables. Thus, we may enter the era of nonradial variability in red giants, where the simple fundamental and overtone pulsation (up to the third and fourth overtone modes) with random effects no longer describe the stars appropriately.

Acknowledgments. We gratefully acknowledge thousands of visual variable star observers around the world whose dedicated efforts over decades and observations made this study possible. This work has been supported by OTKA Grants T022259 and F022249.

\section{References}

Asida, S. M. \& Tuchman, Y. 1995, ApJ, 455, 286

Barnbaum, C., Morris, M., \& Kahane, C. 1995, ApJ, 450, 862

Bedding, T. R., Zijlstra, A. A., Jones, A., \& Foster, G. 1998, MNRAS, 301, 1073

Kiss, L. L., Szatmáry, K., Cadmus, Jr., R. R., \& Mattei, J. A. 1999, A\&A, 346, 542

Xiong, D. R., Ding, L., \& Cheng, Q. L. 1998, ApJ, 499, 355 